\title{
Magnetic excitations and phonons simultaneously studied by resonant inelastic $x$-ray scattering in optimally doped $\mathrm{Bi}_{1.5} \mathrm{~Pb}_{0.55} \mathrm{Sr}_{1.6} \mathrm{La}_{0.4} \mathrm{CuO}_{6+\delta}$
}

\author{
Y. Y. Peng,,${ }^{1, *}$ M. Hashimoto, ${ }^{2}$ M. Moretti Sala, ${ }^{3}$ A. Amorese,${ }^{1, \dagger}$ N. B. Brookes,${ }^{3}$ G. Dellea, ${ }^{1}$ W.-S. Lee,${ }^{4}$ M. Minola,,${ }^{1,}$ \\ T. Schmitt, ${ }^{5}$ Y. Yoshida, ${ }^{6}$ K.-J. Zhou, ${ }^{5,}{ }^{\S}$ H. Eisaki, ${ }^{6}$ T. P. Devereaux, ${ }^{4}$ Z.-X. Shen, ${ }^{4,7}$ L. Braicovich, ${ }^{1,8}$ and G. Ghiringhelli ${ }^{1,8}$ \\ ${ }^{1}$ Dipartimento di Fisica, Politecnico di Milano, Piazza Leonardo da Vinci 32, I-20133 Milano, Italy \\ ${ }^{2}$ Stanford Synchrotron Radiation Lightsource, SLAC National Accelerator Laboratory, 2575, Sand Hill Road, Menlo Park, \\ California 94025, USA \\ ${ }^{3}$ European Synchrotron Radiation Facility (ESRF), BP 220, F-38043 Grenoble Cedex, France \\ ${ }^{4}$ Stanford Institute for Materials and Energy Sciences, SLAC National Accelerator Laboratory, 2575 Sand Hill Road, Menlo Park, \\ California 94025, USA \\ ${ }^{5}$ Swiss Light Source, Paul Scherrer Institut, CH-5232 Villigen PSI, Switzerland \\ ${ }^{6}$ Nanoelectronics Research Institute, AIST, Ibaraki 305-8568, Japan \\ ${ }^{7}$ Geballe Laboratory for Advanced Materials, Departments of Physics and Applied Physics, Stanford University, California 94305, USA \\ ${ }^{8}$ CNR-SPIN, CNISM, Politecnico di Milano, Piazza Leonardo da Vinci 32, I-20133 Milano, Italy \\ (Received 20 April 2015; revised manuscript received 22 June 2015; published 24 August 2015)
}

\begin{abstract}
Magnetic excitations in the optimally doped high- $T_{\mathrm{c}}$ superconductor $\mathrm{Bi}_{1.5} \mathrm{~Pb}_{0.55} \mathrm{Sr}_{1.6} \mathrm{La}_{0.4} \mathrm{CuO}_{6+\delta}(\mathrm{OP}-\mathrm{Bi} 2201$, $T_{\mathrm{c}} \simeq 34 \mathrm{~K}$ ) are investigated by $\mathrm{Cu} L_{3}$ edge resonant inelastic x-ray scattering (RIXS), below and above the pseudogap opening temperature. At both temperatures the broad spectral distribution disperses along the $(1,0)$ direction up to $\sim 350 \mathrm{meV}$ at zone boundary, similar to other hole-doped cuprates. However, above $\sim 0.22$ reciprocal lattice units, we observe a concurrent intensity decrease for magnetic excitations and quasielastic signals with weak temperature dependence. This anomaly seems to indicate a coupling between magnetic, lattice, and charge modes in this compound. We also compare the magnetic excitation spectra near the antinodal zone boundary in the single layer OP-Bi2201 and in the bilayer optimally doped $\mathrm{Bi}_{1.5} \mathrm{~Pb}_{0.6} \mathrm{Sr}_{1.54} \mathrm{CaCu}_{2} \mathrm{O}_{8+\delta}$ $\left(\mathrm{OP}-\mathrm{Bi} 2212, T_{\mathrm{c}} \simeq 96 \mathrm{~K}\right)$. The strong similarities in the paramagnon dispersion and in their energy at zone boundary indicate that the strength of the superexchange interaction and the short-range magnetic correlation cannot be directly related to $T_{\mathrm{c}}$, not even within the same family of cuprates.
\end{abstract}

\section{INTRODUCTION}

In recent years, resonant inelastic X-ray scattering (RIXS) at the $\mathrm{Cu} L_{3}$ edge, thanks to the strong spin-orbit coupling of the $2 p_{3 / 2}$ core-hole intermediate state that provides a direct access to spin flip excitations [1,2], has become a powerful complement to neutron inelastic scattering for the determination of magnetic excitation dispersion in cuprates. The persistence of magnetic excitations has been observed from undoped antiferromagnetic insulators to overdoped superconductors, both for hole- and electron-doped compounds, across the respective superconducting domes [3-8]. This confirms that short-range antiferromagnetic spin correlations survive to high doping and are exceptionally robust. These observations might imply that high energy magnetic fluctuations are necessary for superconductivity, although their possible role as pairing mechanism has not been demonstrated.

Moreover, $\mathrm{Cu} L_{3}$ resonant soft x-ray scattering has decisively contributed to reveal an electronic order now considered ubiquitous in the cuprate superconductors. Earlier

\footnotetext{
*yingying.peng@ polimi.it

${ }^{\dagger}$ Present address: European Synchrotron Radiation Facility (ESRF), BP 220, F-38043 Grenoble Cedex, France.

${ }_{\ddagger}^{\ddagger}$ Present address: Max-Planck-Institut für Festkörperforschung, Heisenbergstraße 1, D-70569 Stuttgart, Germany.

§Present address: Diamond Light Source, Harwell Science and Innovation Campus, Didcot, Oxon, OX11 0DE, United Kingdom.
}

evidence of bulk charge order was observed in La-based cuprates by neutron $[9,10]$ and x-ray scattering [11]. More recently, charge order along the $\mathrm{Cu}-\mathrm{O}$ bond direction has been observed in (Y, Nd) $\mathrm{Ba}_{2} \mathrm{Cu}_{3} \mathrm{O}_{6+x}$ (YBCO, NBCO) [12-16], Bi-based cuprates $\mathrm{Bi}_{2} \mathrm{Sr}_{2-x} \mathrm{La}_{x} \mathrm{CuO}_{6+\delta}$ (Bi2201), and $\mathrm{Bi}_{2} \mathrm{Sr}_{2} \mathrm{CaCu}_{2} \mathrm{O}_{8+\delta}(\mathrm{Bi2212})$ [17-19]. The direct observation of charge order in cuprates came first in the underdoped regime, soon after for the optimal doping of hole-doped cuprates $[19,20]$ and very recently also in the electron-doped compounds [21]. The temperature dependence of charge order in YBCO and Bi2212 displays its competition with superconductivity $[13,15,18]$.

To comprehend the superconductivity of cuprates we need to take into account the diversity of electronic and magnetic excitations. Whether and how these excitations, such as charge, spin, lattice and orbital orders, interact with each other is still the matter of active research. One of the superiority of RIXS is that it can measure different kinds of excitations simultaneously. This allows us to discuss the interplay between the charge, spin, lattice, and orbital orders more in detail within a single experiment. Further, temperature dependence may provide insights into their physical properties and about their role in superconductivity and pseudogap.

In this article we study the collective excitations in the optimally doped $\mathrm{Bi}_{1.5} \mathrm{~Pb}_{0.55} \mathrm{Sr}_{1.6} \mathrm{La}_{0.4} \mathrm{CuO}_{6+\delta}\left(\mathrm{OP}-\mathrm{Bi} 2201, T_{\mathrm{c}} \simeq\right.$ $34 \mathrm{~K})$ by momentum resolved resonant inelastic X-ray scattering (RIXS) at the $\mathrm{Cu} L_{3}$ edge. Considering that the recent angle-resolved photoemission spectroscopy (ARPES) study on OP-Bi2201 showed a particle-hole symmetry breaking 
and a phase transition below the pseudogap temperature $T^{*} \simeq 125 \mathrm{~K}[22,23]$, our measurements were performed at two temperatures, $50 \mathrm{~K}$ (below $T^{*}$ ) and $200 \mathrm{~K}$ (above $\left.T^{*}\right)$. We also compare these results to those of optimally doped $\mathrm{Bi}_{1.5} \mathrm{~Pb}_{0.6} \mathrm{Sr}_{1.54} \mathrm{CaCu}_{2} \mathrm{O}_{8+\delta}\left(\mathrm{OP}-\mathrm{Bi} 2212, T_{\mathrm{c}} \simeq 96 \mathrm{~K}\right)$ to investigate the material dependence of magnetic excitation within the Bi-based superconductor family.

\section{EXPERIMENTAL METHOD}

The high quality OP-Bi2201 and OP-Bi2212 single crystals were grown by the floating zone method. The hole concentration was optimized by annealing the samples in $\mathrm{N}_{2}$ flow. The RIXS experiments for OP-Bi2201 collected at $50 \mathrm{~K}$ and $200 \mathrm{~K}$ were performed with the SAXES instrument [24] at the ADRESS beamline of the Swiss Light Source at the Paul Scherrer Institut [25], the experimental energy resolution was $\sim 150 \mathrm{meV}$; RIXS measurements for OP-Bi2201 and OP-Bi2212 collected at $40 \mathrm{~K}$ were performed with the AXES spectrometer at the beamline ID08 of the European Synchrotron Radiation Facility (ESRF) [26], and the combined energy resolution was $\sim 300 \mathrm{meV}$. The x-ray energy was tuned to the maximum of the $\mathrm{Cu} L_{3}$ absorption peak around $931.3 \mathrm{eV}$. The elastic scattering position was determined with high accuracy for every momentum transfer by comparing the RIXS spectrum to that of policrystalline graphite attached on the sample surface. Samples were cleaved in air some minutes before installation inside the ultrahigh vacuum measurement chamber $\left(\sim 3 \times 10^{-9}\right.$ mbar $)$.

The experimental geometry is shown in Fig. 1(a). X rays are incident on the sample surface at $\theta_{i}$ and scattered by $2 \theta=130^{\circ}$ (constant). The scattering vector $\mathbf{Q}$ is denoted using the pseudotetragonal unit cell with $a=b=3.8 \AA$ and $c=24.4 \AA$ for OP-Bi2201, with $a=b=3.86 \AA$ and $c=31 \AA$ for OP-Bi2212, where the axis $c$ is normal to the cleaved sample surface. $\delta$ is the angle between total momentum $\mathbf{Q}$ and sample $c$ axis. In the experiment $\delta$ is changed by rotating the sample around the vertical axis $b$ in order to change $Q_{\|}$, the projection of the momentum transfer $\mathbf{Q}$ along [100]. Here large negative $Q_{\|}$corresponds to near grazing-incidence geometry; large positive $Q_{\|}$corresponds to near grazing-emission geometry. The $\mathrm{x}$-ray polarization can be chosen parallel $(\pi)$ or perpendicular $(\sigma)$ to the horizontal scattering plane. Figure 1(b) shows the reciprocal space near the Brillouin zone center. The typical size of the Brillouin zone in cuprates is $0.81 \AA^{-1}$ (0.5 reciprocal lattice units, r.l.u.) and the maximum of $Q_{\|}$for $930 \mathrm{eV}$ photons is $0.77 \AA^{-1}$ (0.48 r.l.u.) for $2 \theta=130^{\circ}$. We measured along $( \pm 0.5,0)$ direction and the thick green line represents the region explored in this work. We follow previous conventions [27] and present normalized spectra so that the integrated intensity of the $d d$ excitations $([-3,-1] \mathrm{eV})$ equals one.

\section{RESULTS}

Figure 1(c) displays some RIXS spectra at representative $\delta$ angles for OP-Bi2201, collected at $50 \mathrm{~K}$ and $200 \mathrm{~K}$ with both $\pi$ and $\sigma$ polarized incident $\mathrm{x}$ rays. The spectra exhibit, below $-1.5 \mathrm{eV}, d d$ excitations (transitions of the unpaired hole of $\mathrm{Cu}^{2+}$ from the $d_{x^{2}-y^{2}}$ to other $d$ orbitals) [28-30], with
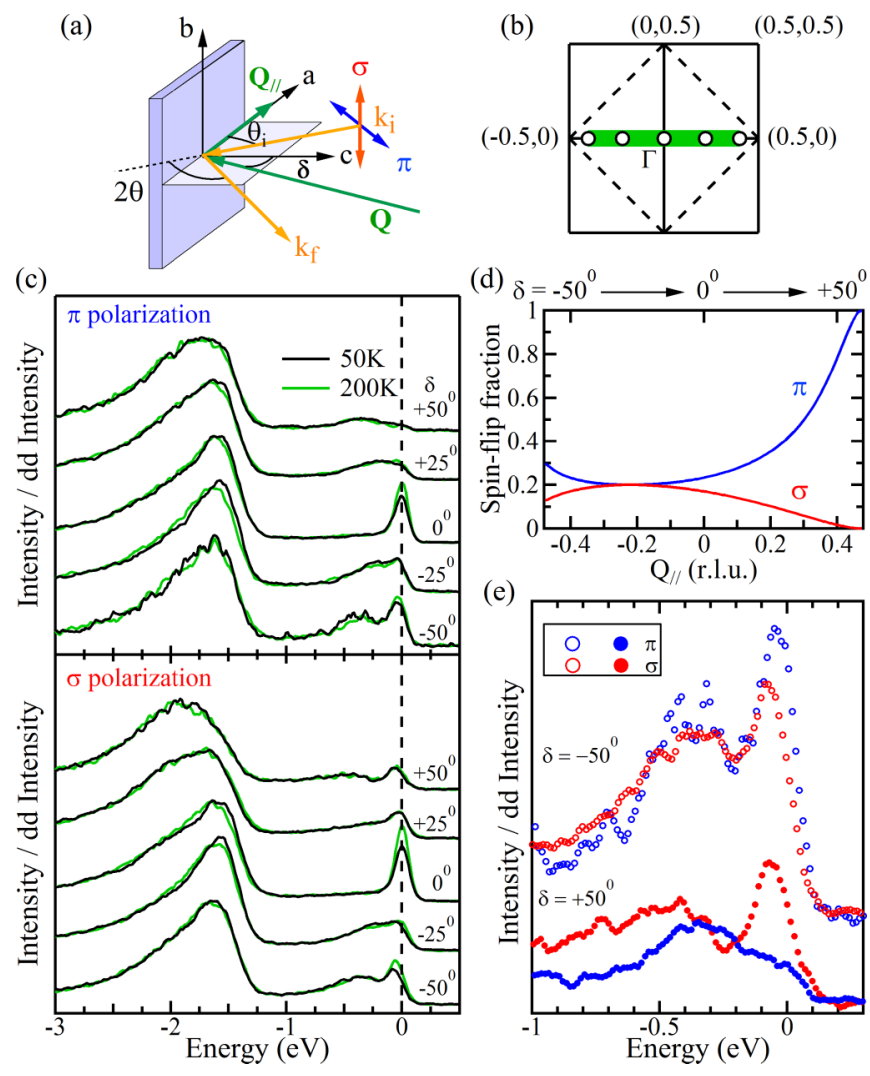

FIG. 1. (Color online) (a) Experimental geometry. (b) Reciprocal-space image, the nuclear and magnetic first Brillouin zones are drawn with solid and dashed lines, respectively; the thick green line indicates the range covered by the experiments. (c) Representative RIXS spectra of OP-Bi2201 for various $\delta$ values in steps of $25^{\circ}$ as indicated by the black empty circles in panel (b), i.e., from $15^{\circ}$ grazing incidence to $15^{\circ}$ grazing emission, as measured with respect to the sample surface. Top: $\pi$ polarized incident $\mathrm{x}$ rays. Bottom: $\sigma$ polarized incident $\mathrm{x}$ rays. Data were collected at $50 \mathrm{~K}$ and $200 \mathrm{~K}$. (d) Single spin-flip fraction (without orbital excitation) for $\pi$ or $\sigma$ polarizations according to RIXS cross section calculations for $2 \theta=130^{\circ}$. The non-spin-flip channel can be spread over elastic, charge excitations, double-spin-flip excitations, and phonons, covering a broad spectral range from 0 to $1 \mathrm{eV}$ energy loss. This explains why spin-flip channel, less intense but more concentrated in energy, is often the most easily recognizable spectral feature. (e) RIXS spectra measured at $Q_{\|}=-0.4$ r.l.u. with grazingincidence geometry $\left(\delta=-50^{\circ}\right)$ and measured at $Q_{\|}=0.4$ r.l.u. with grazing-emission geometry $\left(\delta=+50^{\circ}\right)$ for $\pi$ - or $\sigma$-polarized light. Data were collected at $50 \mathrm{~K}$. All spectra are normalized to the integrated intensity of the $d d$ excitations.

no obvious temperature dependence. Two broad peaks around $-1.6 \mathrm{eV}$ and around $-1.9 \mathrm{eV}$ can be roughly ascribed to the transitions to the $d_{x y}$ and $d_{x z / y z}$ orbitals, in analogy to the results obtained in the undoped compounds by Moretti Sala et al. [28]. The $d_{3 z^{2}-r^{2}}$ final state, more difficult to discern, is probably at slightly higher energy loss. The elastic peak is, as usual, stronger at specular angle $\left(\delta=0^{\circ}\right)$ due to the reflectivity from the surface. The dispersion of the $d d$ excitations is as small as that observed previously in other layered cuprates [28] within the energy resolution of our experiment. 
In layered cuprates the simplest magnetic excitation implies the reversal of the spin $1 / 2$ at one site, giving origin to a magnon in the antiferromagnetic parent compounds and to a so-called paramagnon in doped materials. In all cases the single spin-flip excitation is obtained through the rotation by $90^{\circ}$ of the scattering photon polarization vector: it turns out that the only $\sigma \pi^{\prime}$ and $\pi \sigma^{\prime}$ combinations lead to nonzero spin-flip cross section, where the prime denotes the polarization of the scattered photon [1,31], because due to the $x^{2}-y^{2}$ symmetry of the $3 d$ hole, the relevant polarization rotation is that projected on the $a b$ plane. In Fig. 1(d) we show the spin-flip fraction as calculated with the simplified RIXS cross sections of Refs. $[1,27,28]$ for $2 \theta=130^{\circ}$. The blue (red) line represents the spin-flip fraction of the low energy excitation spectral weight for incident $\pi(\sigma)$ polarization. For incident $\pi$ polarization at positive $Q_{\|}$, most of the low-energy signal originates from single spin-flip, with a small fraction arising from the non-spin-flip channel. All other scattering geometries probe a mix of spin-flip and non-spin events without analysis of the scattered photon polarization. These incident polarization dependences have been supported by numerical calculations [31,32], and have been further confirmed by a recent RIXS experiment obtained by using a soft $\mathrm{x}$-ray polarimeter capable of measuring the polarization of the scattered radiation [33,34] simultaneously with the spectral distribution. It should be kept in mind that, whereas the non-spin-flip part is spread over several contributions differing in nature (charge excitations, phonons, diffuse elastic, double spin-flip) and energy, the single spin-flip channel is concentrated in the resolution limited peak in the antiferromagnetic parent compounds or in the (often broad) paramagnon in the doped superconductors. This fact makes the spin-flip excitations more evident and recognizable with respect to the non-spin-flip excitations. This is experimentally demonstrated in Fig. 1(e). At $\delta=-50^{\circ}$ for both polarizations the spectra display a broad distribution in the midinfrared region, provided by a combination of single and multiple paramagnons, charge and vibrational excitations, all difficult to disentangle. This confirms that, at large negative $\delta$ values, the single spin-flip fraction is relative small for both incident polarizations. On the other hand, at large positive $\delta$ values, single spin-flip excitations are prominent for $\pi$ and suppressed for $\sigma$ polarization. This is evident in the spectra at $\delta=+50^{\circ}$ : for incident $\pi$ polarization, it shows a peak at $\sim 350 \mathrm{meV}$ which is a single spin-flip excitation according to above discussions; for incident $\sigma$ polarization, it shows a higher peak at $\sim 400 \mathrm{meV}$, which is a well recognizing bimagnon feature [32].

In Fig. 2 we show the evolution of the spin-flip spectral component along the $(0,0)-(0.5,0)$ symmetry direction. It appears from the raw data that the paramagnon mode disperses to high energy loss similarly to that of other superconducting cuprates [4-7]. We decompose the spectra into four different contributions [5,6]: a resolution-limited Gaussian for the elastic peak, an antisymmetrized Lorentzian for the magnetic scattering, a smooth background for the particle-hole continuum and the tail of $d d$ excitations, and a resolution-limited Gaussian for the dominant optical phonon mode of $\sim 65 \mathrm{meV}$. Around this energy previous Raman [35] and high-resolution inelastic x-ray scattering [36] have reported optical phonon mode with strong electron-phonon

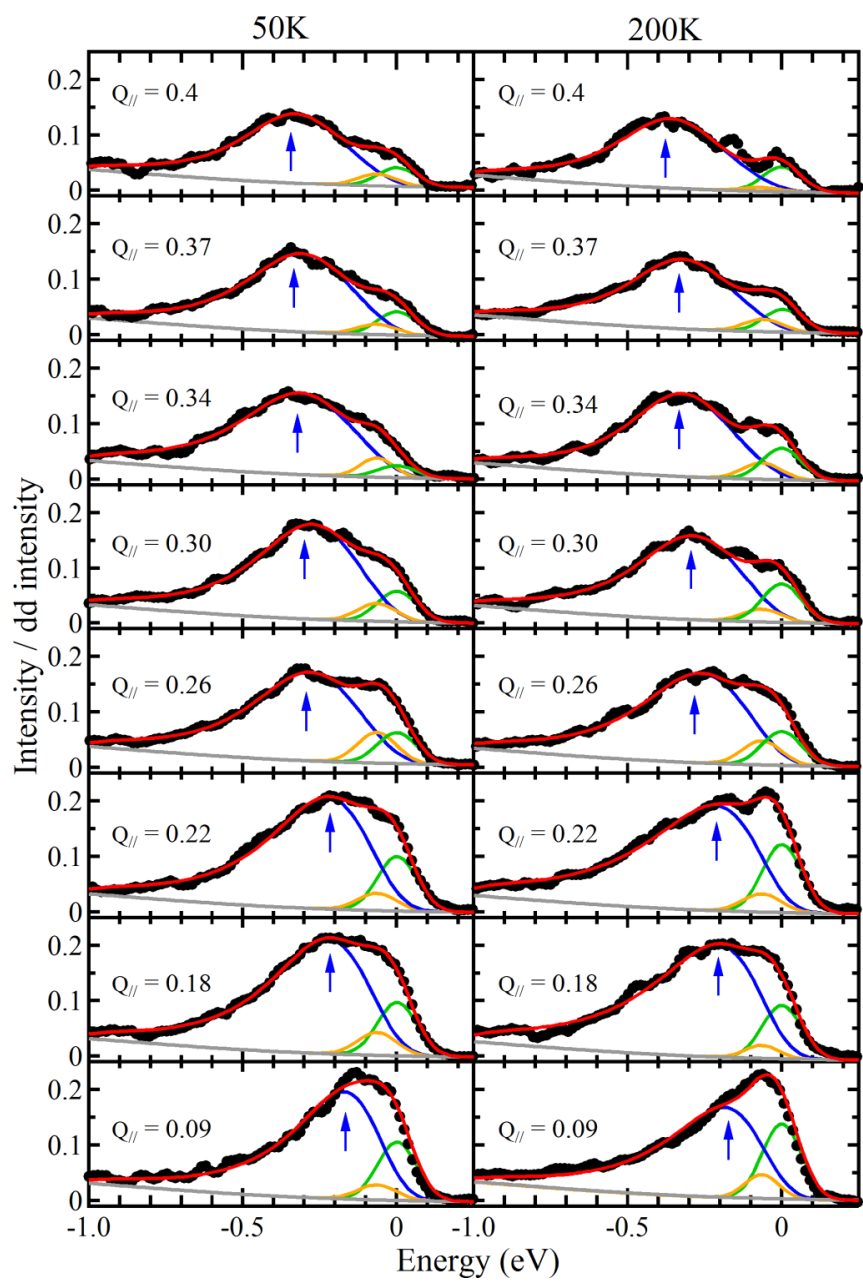

FIG. 2. (Color online) RIXS spectra of OP-Bi2201 along (0,0)$(0.5,0)$ symmetry direction (black solid circles) at $50 \mathrm{~K}$ (left) and $200 \mathrm{~K}$ (right). The spectra are decomposed into the dispersive magnetic excitations (blue line), the elastic scattering (green line), the phonon scattering (orange line), and the charge- and $d d$-excitations background (gray line). The blue arrow indicates the paramagnon peak. The data were collected at grazing-emission using $\pi$ incident polarization.

coupling. Its strong coupling with electrons, also manifested in ARPES by a kink in the electronic state dispersion [36,37], is significant in RIXS. Within the present experimental accuracy we can not determine the phonon dispersion, and the crucial implications of its observation in $\mathrm{Cu} L_{3}$ RIXS will be discussed elsewhere [38].

We track the paramagnon peak as denoted by the blue arrows in Fig. 2, and superimpose it on the energy/momentum intensity map in Fig. 3 for (a) $50 \mathrm{~K}$ (below $T^{*}$ ) and (b) $200 \mathrm{~K}$ (above $T^{*}$ ). To study the paramagnon we focus on the intensity map at positive $Q_{\|}$, where the spin-flip dominates the lowenergy excitations as explained previously. From the maps we note that the intensity of magnetic excitations shows a decrease above $Q_{\|} \simeq 0.22$ r.l.u. for both temperatures; meanwhile one feature appears below $100 \mathrm{meV}$ at $Q_{\|} \simeq 0.22$ r.l.u., which will be discussed subsequently. To visualize if there is any temperature dependence, we overlap the raw spectra measured at the two temperatures in Fig. 3(c). The two sets of spectra 

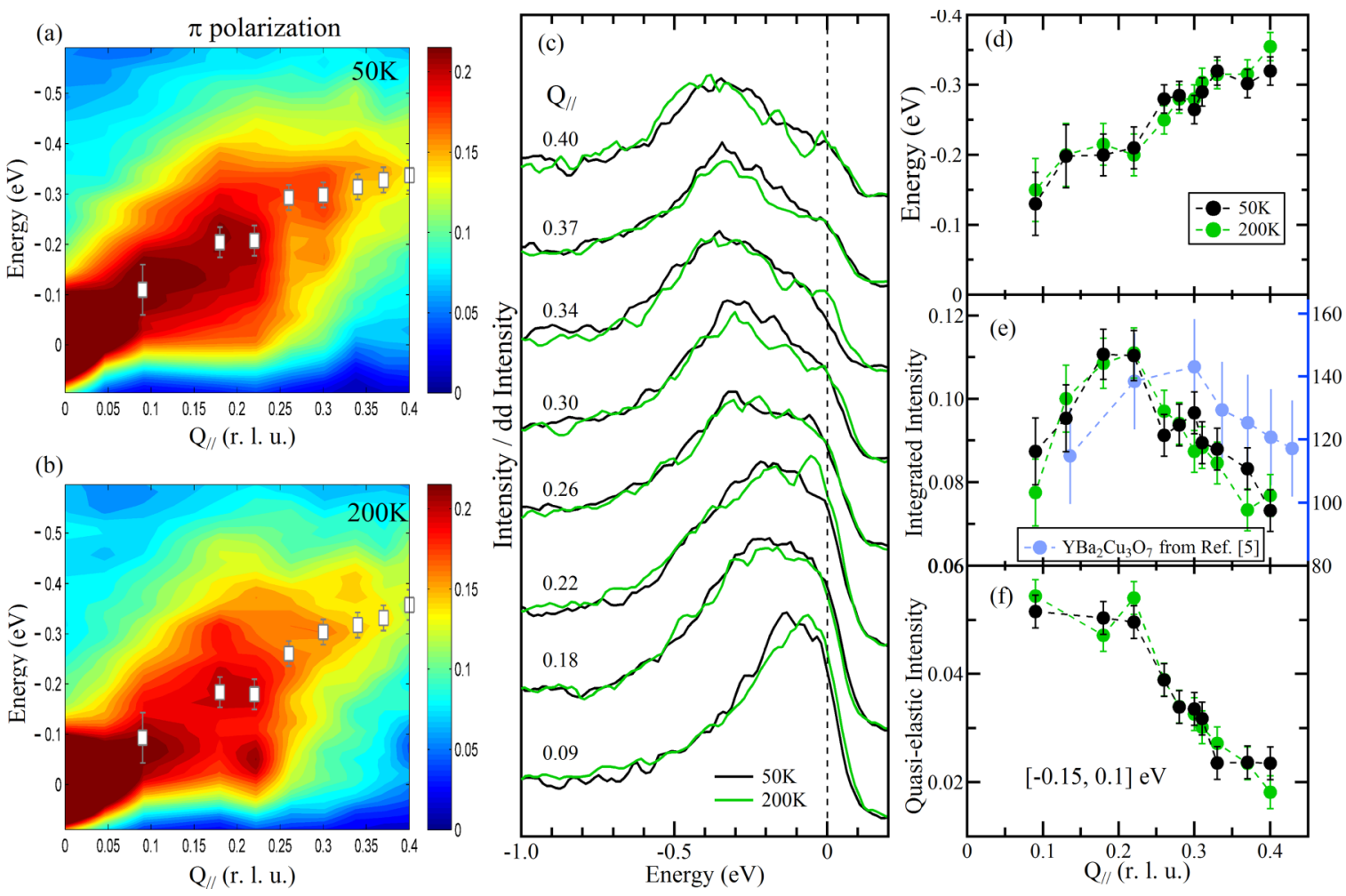

FIG. 3. (Color online) Energy/momentum intensity false-color maps of RIXS spectra along $(0,0)-(0.5,0)$ symmetry direction measured at (a) $50 \mathrm{~K}$ and (b) $200 \mathrm{~K}$ with $\pi$ polarization on OP-Bi2201. The white solid squares indicate the paramagnon peak positions as determined with the fitting procedure illustrated in Fig. 2. (c) RIXS spectra at $50 \mathrm{~K}$ (black) and $200 \mathrm{~K}$ (green) at selected $Q_{\|}$. (d) Experimental paramagnon dispersion and (e) the integrated intensity of paramagnon peak at $50 \mathrm{~K}$ (black) and $200 \mathrm{~K}$ (green) determined from the fitting procedure. Integrated inelastic intensity of optimally doped $\mathrm{YBa}_{2} \mathrm{Cu}_{3} \mathrm{O}_{7}$ from Ref. [5] is superimposed for comparison (blue). (f) Intensity at [ $\left.-0.15,0.1\right] \mathrm{eV}$ energy window for quasielastic signal at $50 \mathrm{~K}$ (black) and $200 \mathrm{~K}$ (green). Self-absorption correction has been applied to (e) and (f). The error bars represent the uncertainty in the fitting.

are almost identical within the experimental uncertainty, confirming therefore that the effective superexchange interaction $J_{\text {eff }}$ is very weakly temperature dependent [39].

The energy and integrated intensity of paramagnon determined from the fitting procedure are compared respectively in Figs. 3(d) and 3(e) for the two temperatures. In the antiferromagnetic systems the paramagnon intensity is expected theoretically to decrease to zero when approaching the $\Gamma$ point [1,2]. From Fig. 3(e) we observe a similar behavior below 0.2 r.l.u. also in OP-Bi2201, although the paramagnon intensity could not be tracked below 0.1 r.l.u. due to the onset, towards $\Gamma$, of the elastic peak that hinders any possible sub-100 meV inelastic feature with the present resolution. More interestingly though, the intensity of paramagnon also shows an abrupt drop above $Q_{\|} \simeq 0.22$ r.l.u. In Fig. 3(f) we integrated over $[-0.15,0.1] \mathrm{eV}$ energy window to evaluate the quasielastic intensity: the intensity shows a step (or a peak) at $Q_{\|} \simeq 0.22$ r.l.u. and then decreases with momentum for both temperatures. We have applied self-absorption correction [34] to the intensity and found that the change of intensity is less than $10 \%$ near the Brillouin zone boundary, which cannot explain the strong depression of intensity above 0.22 r.l.u. here (nearly $40 \%$ decrease around the Brillouin zone boundary). For bismuth cuprates the small correction for the spectra normalized to $d d$ intensity is due to the relative large preedge signal of x-ray absorption spectra, originated from the large nonresonant absorption of bismuth. The coincident intensity drop of paramagnon and quasielastic signal indicates a nontrivial effect. We notice that for optimally doped $\mathrm{YBa}_{2} \mathrm{Cu}_{3} \mathrm{O}_{7}$ in Refs. [5], the integrated inelastic intensity shows a decrease above 0.3 r.l.u., a wave vector characterizing charge order [20] and bond-buckling phonon anomaly [40]. Therefore, charge order and phonon anomaly might play a role in our OP-Bi2201 too, as discussed below. We also notice that around $Q_{\|} \simeq 0.22$ r.l.u. the paramagnon energy in Fig. 3(d) seems to deviate from a smooth dispersion, which deserves further investigation as well.

\section{DISCUSSION}

\section{A. Intensity maps of RIXS spectra}

The concurrent intensity drop of paramagnon and quasielastic signal around $Q_{\|}=0.22$ r.l.u. are probably not a fortuitous coincidence. In the map of Fig. 3(b), we can directly observe a feature below $100 \mathrm{meV}$ at $Q_{\|} \simeq 0.22$ r.l.u. This can be put in relation with other intriguing phenomena taking place in Bi2201, such as charge order and strong electron-phonon coupling. Charge order, with $Q_{\mathrm{CO}}=0.243$ r.l.u., has been observed recently in underdoped Bi2201 $\left(T_{\mathrm{c}}=30 \mathrm{~K}\right)$ by resonant x-ray scattering [17] and, with $Q_{\|} \sim 0.28$ r.l.u., in OP-Bi2212 by RIXS [19]. Here the wave vector of the low energy feature, i.e., 0.22 r.l.u., is compatible with the one of 

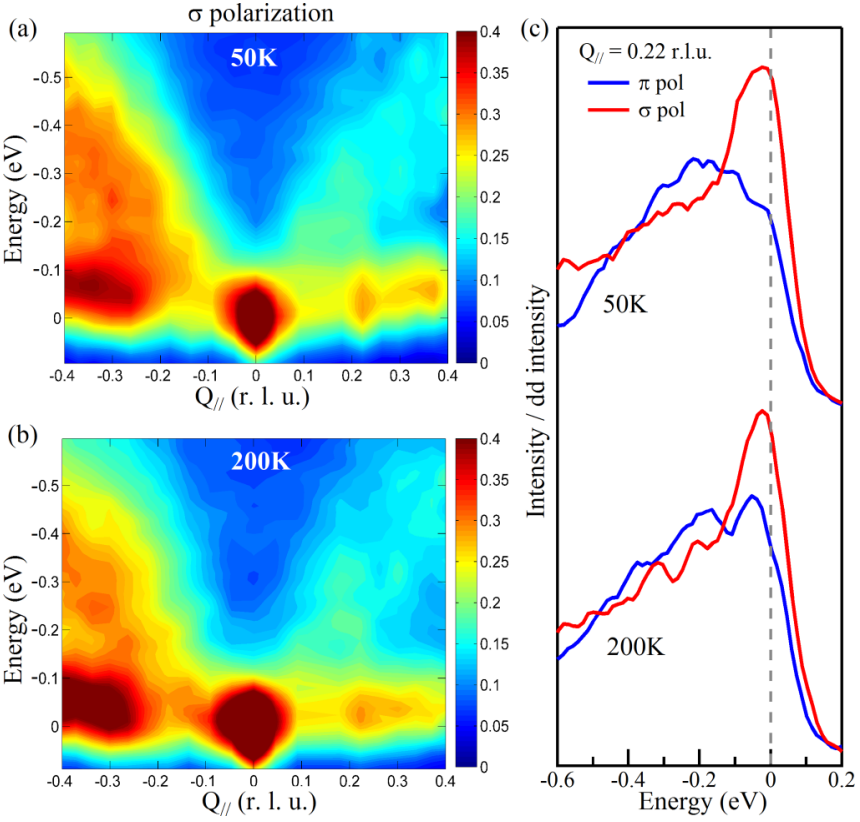

FIG. 4. (Color online) Energy/momentum intensity false-color maps of RIXS spectra along $(-0.5,0)-(0.5,0)$ symmetry direction measured at (a) $50 \mathrm{~K}$ and (b) $200 \mathrm{~K}$ with $\sigma$ polarization on OP-Bi2201. (c) Polarization comparison of RIXS spectra measured at $Q_{\|}=0.22$ r.l.u. at $50 \mathrm{~K}$ and $200 \mathrm{~K}$.

a charge order signal. However, the persistence of the feature up to $200 \mathrm{~K}$, above the presumed charge order temperature, seems in contrast with the commonly observed temperature dependence of the charge order in hole-doped cuprates [13,17]. In the present RIXS data on OP-Bi2201 we did not find an evidence for charge order, possibly because of the excessive disorder, known to be higher in Bi2201 than in Bi2212 [18,41]. Unfortunately the lack of an unambiguous evidence of charge order in the present sample does not allow us to make a direct connection, on that issue, to the ARPES results, which showed particle-hole symmetry breaking [22] and a phase transition below the pseudogap temperature [23]. On the other hand, we can exploit the richness of the RIXS spectra.

The assignment of the low energy feature can be inspired by observing the RIXS colormaps of Figs. 4(a) and 4(b), where the $\sigma$ polarization was used. At positive $Q_{\|}$the $\sigma$ polarization enhances the non-spin-flip final states, including bimagnonlike magnetic excitations, particle-hole pair generation and phonons; at negative $Q_{\|}$the spin-flip and non-spin-flip excitations have similar intensity. We can unambiguously observe a peak below $100 \mathrm{meV}$ at $Q_{\|}=+0.22$ r.l.u.: this peak is stronger with $\sigma$ than $\pi$ polarization, as highlighted by the spectra comparison in Fig. 4(c), a clear demonstration of its non-spin-flip character. Its energy is fully compatible with a phonon excitation. This assignment is confirmed by the data at negative $Q_{\|}$, where the phonon peak has an almost flat dispersion but a clear increase in intensity beyond -0.25 r.l.u. Interestingly, inelastic $\mathrm{x}$-ray scattering measurement on optimally doped $\mathrm{Bi}_{2} \mathrm{Sr}_{1.6} \mathrm{La}_{0.4} \mathrm{CuO}_{6+\delta}$ found that the $\mathrm{Cu}-\mathrm{O}$ bond stretching (BS) phonon shows a softening and an anomalously broad line shape around $0.22-0.25$ r.l.u. [36]. The crossing of two longitudinal phonon modes and/or the anomalous broadening of the BS phonon may have a relation to the strong phonon signal around 0.22 r.l.u. in RIXS. Moreover, phonon softening and broadening has been observed around the charge ordering wave vector in several copper oxide superconductors $[40,42,43]$, revealing the correlation between charge order and phonon anomalies in experiments other than RIXS. What RIXS is adding here in the specific case of OP$\mathrm{Bi} 2201$ is the coincidence of phonon and paramagnon intensity anomalies, appearing in the putative charge-order wave-vector region. Interestingly enough, optimally doped YBCO shows a similar maximum of the paramagnon intensity around its own charge-order wave vector (0.30 r.l.u.), as shown in Fig. 3(e). The $q$ dependence of the phonon intensity in RIXS and its relation with charge order goes beyond the scope of this article and will be treated more systematically elsewhere [38]. Moreover, in the near future better resolved and more systematic RIXS measurements will help to clarify the connection of charge order and phonon anomaly with the paramagnon energy and intensity evolution.

\section{B. Relation between $J_{\text {eff }}$ and $T_{\mathrm{c}}$}

In Fig. 5 we compare the RIXS spectra of OP-Bi2201 and OP-Bi2212 at large $Q_{\|}$for $\pi$ polarization. In panel (a), the two data sets for OP-Bi2201 (one at $50 \mathrm{~K}$ with $150 \mathrm{meV}$ resolution; one at $40 \mathrm{~K}$ with $300 \mathrm{meV}$ resolution) are compatible with each other, once the difference in resolution is taken into account. For OP-Bi2212 (40 K, $300 \mathrm{meV}$ resolution) we observe that the $d d$ multiplet is more extended towards higher energy, due to the $d_{3 z^{2}-r^{2}}$ final state being more separated from the $d_{x^{2}-y^{2}}$, due to the absence of one apical oxygen in the bilayer compound with respect to the single layer $\mathrm{Bi} 2201$. Panel (b) shows an enlarged view of the low energy portion. The paramagnon energies are similar in the two samples. Following the usual fitting procedure [5,6], we obtain that the paramagnon energy at zone boundary is $\sim 350 \mathrm{meV}$, as shown in Fig. 5(c). At small $Q_{\|}$the fitting for spectra with lower resolution is too uncertain and we cannot plot the corresponding points in panel (c). We present the integrated intensities for OP-Bi2201 and OP-Bi2212 in Fig. 5(d), which are very similar with a consistent drop above $\sim 0.22$ r.l.u., suggesting a common interplay in the Bi-based cuprate family.

These results are in stark contrast with those recently published by Dean et al. [44], who have found that the paramagnon energy at zone boundary is substantially higher in $\mathrm{Bi}_{2} \mathrm{Sr}_{2} \mathrm{Ca}_{2} \mathrm{Cu}_{3} \mathrm{O}_{10+\delta}\left(\mathrm{Bi}-2223, T_{\mathrm{c}}=109 \mathrm{~K}\right)$ than in $\mathrm{Bi}_{2+x} \mathrm{Sr}_{2-x} \mathrm{CuO}_{6+\delta}\left(\mathrm{Bi}-2201, T_{\mathrm{c}} \simeq 1 \mathrm{~K}\right)$. In Ref. [44] the paramagnon energies are assigned at $295 \mathrm{meV}$ for $\mathrm{Bi}-2201$ and $347 \mathrm{meV}$ for $\mathrm{Bi}-2223$ close to $(1 / 2,0, L)$. From this result the authors argued that $T_{\mathrm{c}}$ scales monotonically with

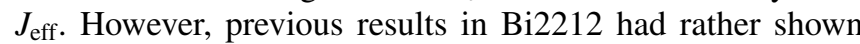
an opposite trend, i.e., the softening with doping of the magnetic excitation energy, namely from $\sim 350 \mathrm{meV}$ in the heavily underdoped nonsuperconducting sample to $\sim 300 \mathrm{meV}$ in the optimally doped one $\left(T_{\mathrm{c}}=92 \mathrm{~K}\right)$ [7]. Here we find that the paramagnon energies of OP-Bi2201 $\left(T_{\mathrm{c}} \simeq 34 \mathrm{~K}\right)$ and OP-Bi2212 $\left(T_{\mathrm{c}} \simeq 96 \mathrm{~K}\right)$ are indeed similar to that of $\mathrm{Bi}-2223$ $\left(T_{\mathrm{c}}=109 \mathrm{~K}\right)$ and of heavily underdoped Bi2212. Therefore, our results confirm for the $\mathrm{Bi} 22 \mathrm{~nm}$ family what was already known for YBCO and LSCO [5,6,8], that the value of the 

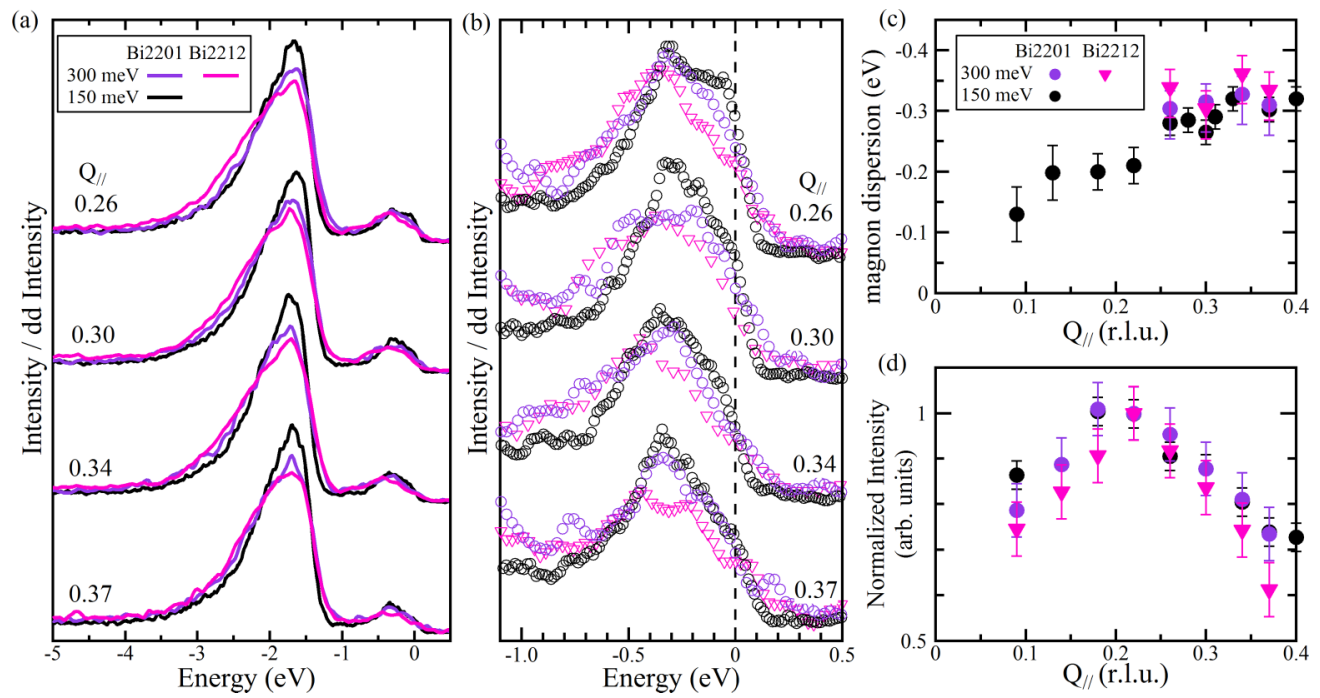

FIG. 5. (Color online) (a) Comparison of RIXS spectra of OP-Bi2201 and OP-Bi2212 at large $Q_{\|}$measured with $\pi$ polarization. Two data sets were collected for OP-Bi2201, at $50 \mathrm{~K}$ and $40 \mathrm{~K}$ with energy resolution of $150 \mathrm{meV}$ (black) and $300 \mathrm{meV}$ (purple), respectively; OP-Bi2212 data were collected at $40 \mathrm{~K}$ with energy resolution of $300 \mathrm{meV}$ (magenta). (b) Enlarged view of the low energy portion. (c) The paramagnon energies of OP-Bi2201 and OP-Bi2212 from the fitting of the spectra with experimental resolution of 300 meV are overlaid with paramagnon energy of OP-Bi2201 at $50 \mathrm{~K}$ reproduced from Fig. 3(d). The large error bars are due to the fitting uncertainty for the lower resolution spectra. (d) Integrated intensities of RIXS spectra with energy window $[-0.8,0] \mathrm{eV}$, normalized to the respective value at $Q_{\|}=0.22$ r.l.u. Self-absorption correction has been applied to the intensities. The error bars represent the uncertainty in determining the spectral weight.

magnetic excitation energy at zone boundary does not correlate directly to $T_{\mathrm{c}}$. The exception found in Ref. [44] is possibly due to the different local structure $(\mathrm{Cu}-\mathrm{O}$ distance; $\mathrm{Cu}-\mathrm{O}-\mathrm{Cu}$ bond angle) of the Bi-doped compound, chosen for its especially low $T_{\mathrm{c}}$ at optimal doping. Although there is a good probability that magnetic fluctuations play a decisive role in high- $T_{\mathrm{c}}$ superconductivity, the maximum of $T_{\mathrm{c}}$ for different materials may be more strongly influenced by other factors than just the value of the superexchange coupling.

\section{CONCLUSIONS}

In the RIXS spectra measured with the appropriate conditions ( $\pi$ polarization, positive $Q_{\|}$values), we have observed the simultaneous intensity decrease of the paramagnon and the quasielastic signal above $\sim 0.22$ r.l.u., the wave vector of the putative charge order in OP-Bi2201. The tiny temperature dependence and the evidence of a concurrent onset, with $\sigma$ polarization, of the phonon signal at that special wave vector, hint at a combined effect, on the magnetic excitation spectrum, of electron-phonon coupling and incipient charge order. Further insight into this issue could come from considering the influence of phonon and charge order on the spin dynamical structure factor. Moreover, we give here a further confirmation of the robustness of magnetic excitations across the phase diagram of high $T_{\mathrm{c}}$ cuprate superconductors. There is, however, an increasing general evidence that, in cuprates, spin excitations get coupled, via the electron-phonon interaction, to both lattice modes and charge order, therefore providing a ubiquitous ingredient for the superconductivity pairing mechanisms. A better clarification of this three-actors scenario (spin excitations, electron-phonon coupling, and charge order) will require further systematic use of high resolution resonant elastic and inelastic x-ray scattering.

\section{ACKNOWLEDGMENTS}

The spectra at the ADRESS beam line of the Swiss Light Source were measured using the SAXES instrument developed jointly by Paul Scherrer Institut (Villigen, Switzerland), Politecnico di Milano (Italy) and École Polytechnique Fédérale de Lausanne (Switzerland). This work is supported by the PIK project POLARIX of the Italian Ministry of Research (MIUR).
[1] L. J. P. Ament, G. Ghiringhelli, M. M. Sala, L. Braicovich, and J. van den Brink, Phys. Rev. Lett. 103, 117003 (2009).

[2] M. W. Haverkort, Phys. Rev. Lett. 105, 167404 (2010).

[3] K. Ishii, M. Fujita, T. Sasaki, M. Minola, G. Dellea, C. Mazzoli, K. Kummer, G. Ghiringhelli, L. Braicovich, T. Tohyama, K. Tsutsumi, K. Sato, R. Kajimoto, K. Ikeuchi, K. Yamada, M. Yoshida, M. Kurooka, and J. Mizuki, Nature Commun. 5, 3714 (2014).
[4] L. Braicovich, J. van den Brink, V. Bisogni, M. M. Sala, L. J. P. Ament, N. B. Brookes, G. M. De Luca, M. Salluzzo, T. Schmitt, V. N. Strocov, and G. Ghiringhelli, Phys. Rev. Lett. 104, 077002 (2010).

[5] M. Le Tacon, G. Ghiringhelli, J. Chaloupka, M. M. Sala, V. Hinkov, M. W. Haverkort, M. Minola, M. Bakr, K. J. Zhou, S. Blanco-Canosa, C. Monney, Y. T. Song, G. L. Sun, C. T. Lin, G. M. De Luca, M. Salluzzo, G. Khaliullin, 
T. Schmitt, L. Braicovich, and B. Keimer, Nat. Phys. 7, 725 (2011).

[6] M. P. M. Dean, G. Dellea, R. S. Springell, F. Yakhou-Harris, K. Kummer, N. B. Brookes, X. Liu, Y.-J. Sun, J. Strle, T. Schmitt, L. Braicovich, G. Ghiringhelli, I. Bozovic, and J. P. Hill, Nat. Mater. 12, 1019 (2013).

[7] M. P. M. Dean, A. J. A. James, R. S. Springell, X. Liu, C. Monney, K. J. Zhou, R. M. Konik, J. S. Wen, Z. J. Xu, G. D. Gu, V. N. Strocov, T. Schmitt, and J. P. Hill, Phys. Rev. Lett. 110, 147001 (2013).

[8] M. Le Tacon, M. Minola, D. C. Peets, M. Moretti Sala, S. BlancoCanosa, V. Hinkov, R. Liang, D. A. Bonn, W. N. Hardy, C. T. Lin, T. Schmitt, L. Braicovich, G. Ghiringhelli, and B. Keimer, Phys. Rev. B 88, 020501 (2013).

[9] J. M. Tranquada, B. J. Sternlieb, J. D. Axe, Y. Nakamura, and S. Uchida, Nature (London) 375, 561 (1995).

[10] M. Fujita, H. Goka, K. Yamada, and M. Matsuda, Phys. Rev. Lett. 88, 167008 (2002).

[11] P. Abbamonte, A. Rusydi, S. Smadici, G. D. Gu, G. A. Sawatzky, and D. L. Feng, Nat. Phys. 1, 155 (2005).

[12] T. Wu, H. Mayaffre, S. Krämer, M. Horvatic, C. Berthier, W. N. Hardy, R. X. Liang, D. A. Bonn, and M.-H. Julien, Nature (London) 477, 191 (2011).

[13] G. Ghiringhelli, M. Le Tacon, M. Minola, S. Blanco-Canosa, C. Mazzoli, N. B. Brookes, G. M. De Luca, A. Frano, D. G. Hawthorn, F. He, T. Loew, M. M. Sala, D. C. Peets, M. Salluzzo, E. Schierle, R. Sutarto, G. A. Sawatzky, E. Weschke, B. Keimer, and L. Braicovich, Science 337, 821 (2012).

[14] A. J. Achkar, R. Sutarto, X. Mao, F. He, A. Frano, S. Blanco-Canosa, M. Le Tacon, G. Ghiringhelli, L. Braicovich, M. Minola, M. Moretti Sala, C. Mazzoli, R. Liang, D. A. Bonn, W. N. Hardy, B. Keimer, G. A. Sawatzky, and D. G. Hawthorn, Phys. Rev. Lett. 109, 167001 (2012).

[15] J. Chang, E. Blackburn, A. T. Holmes, N. B. Christensen, J. Larsen, J. Mesot, R. Liang, D. A. Bonn, W. N. Hardy, A. Watenphul, M. v. Zimmermann, E. M. Forgan, and S. M. Hayden, Nat. Phys. 8, 871 (2012).

[16] E. Blackburn, J. Chang, M. Hucker, A. T. Holmes, N. B. Christensen, R. Liang, D. A. Bonn, W. N. Hardy, U. Rutt, O. Gutowski, M. v. Zimmermann, E. M. Forgan, and S. M. Hayden, Phys. Rev. Lett. 110, 137004 (2013).

[17] R. Comin, A. Frano, M. M. Yee, Y. Yoshida, H. Eisaki, E. Schierle, E. Weschke, R. Sutarto, F. He, A. Soumyanarayanan, Y. He, M. Le Tacon, I. Elfimov, J. E. Hoffman, G. Sawatzky, B. Keimer, and A. Damascelli, Science 343, 390 (2014).

[18] E. H. da Silva Neto, P. Aynajian, A. Frano, R. Comin, E. Schierle, E. Weschke, A. Gyenis, J. S. Wen, J. Schneeloch, Z. J. Xu, S. Ono, G. D. Gu, M. Le Tacon, and A. Yazdani, Science 343, 393 (2014).

[19] M. Hashimoto, G. Ghiringhelli, W.-S. Lee, G. Dellea, A. Amorese, C. Mazzoli, K. Kummer, N. B. Brookes, B. Moritz, Y. Yoshida, H. Eisaki, Z. Hussain, T. P. Devereaux, Z.-X. Shen, and L. Braicovich, Phys. Rev. B 89, 220511(R) (2014).

[20] S. Blanco-Canosa, A. Frano, E. Schierle, J. Porras, T. Loew, M. Minola, M. Bluschke, E. Weschke, B. Keimer, and M. Le Tacon, Phys. Rev. B 90, 054513 (2014).

[21] E. H. da Silva Neto, R. Comin, F. He, R. Sutarto, Y. Jiang, R. L. Greene, G. A. Sawatzky, and A. Damascelli, Science 347, 6219 (2015).
[22] M. Hashimoto, R.-H. He, K. Tanaka, J.-P. Testaud, W. Meevasana, R. G. Moore, D. H. Lu, H. Yao, Y. Yoshida, H. Eisaki, T. P. Devereaux, Z. Hussain, and Z.-X. Shen, Nat. Phys. 6, 414 (2010).

[23] R.-H. He, M. Hashimoto, H. Karapetyan, J. D. Koralek, J. P. Hinton, J. P. Testaud, V. Nathan, Y. Yoshida, Hong Yao, K. Tanaka, W. Meevasana, R. G. Moore, D. H. Lu, S.-K. Mo, M. Ishikado, H. Eisaki, Z. Hussain, T. P. Devereaux, S. A. Kivelson, J. Orenstein, A. Kapitulnik, and Z.-X. Shen, Science 331, 1579 (2011).

[24] G. Ghiringhelli, A. Piazzalunga, C. Dallera, G. Trezzi, L. Braicovich, T. Schmitt, V. N. Strocov, R. Betemps, L. Patthey, X. Wang, and M. Grioni, Rev. Sci. Instrum. 77, 113108 (2006).

[25] V. N. Strocov, T. Schmitt, U. Flechsig, T. Schmidt, A. Imhof, Q. Chen, J. Raabe, R. Betemps, D. Zimoch, J. Krempasky, X. Wang, M. Grioni, A. Piazzalunga, and L. Patthey, J. Synchrotron Radiat. 17, 631 (2010).

[26] C. Dallera, E. Puppin, G. Trezzi, N. Incorvaia, A. Fasana, L. Braicovich, N. B. Brookes, and J. B. Goedkoop, J. Synchrotron Radiat. 3, 231 (1996).

[27] L. Braicovich, M. Moretti Sala, L. J. P. Ament, V. Bisogni, M. Minola, G. Balestrino, D. Di Castro, G. M. De Luca, M. Salluzzo, G. Ghiringhelli, and J. van den Brink, Phys. Rev. B 81, 174533 (2010).

[28] M. Moretti Sala, V. Bisogni, C. Aruta, G. Balestrino, H. Berger, N. B. Brookes, G. M. de Luca, D. Di Castro, M. Grioni, M. Guarise, P. G. Medaglia, F. Miletto Granozio, M. Minola, P. Perna, M. Radovic, M. Salluzzo, T. Schmitt, K. J. Zhou, L. Braicovich, and G. Ghiringhelli, New J. Phys. 13, 043026 (2011).

[29] M. Minola, D. Di Castro, L. Braicovich, N. B. Brookes, D. Innocenti, M. Moretti Sala, A. Tebano, G. Balestrino, and G. Ghiringhelli, Phys. Rev. B 85, 235138 (2012).

[30] L. Hozoi, L. Siurakshina1, P. Fulde, and J. van den Brink, Sci. Rep. 1, 65 (2011).

[31] C. J. Jia, E. A. Nowadnick, K. Wohlfeld, Y. F. Kung, C.-C. Chen, S. Johnston, T. Tohyama, B. Moritz, and T. P. Devereaux, Nature Commun. 5, 3314 (2014).

[32] J. I. Igarashi and T. Nagao, Phys. Rev. B 85, 064421 (2012).

[33] L. Braicovich, M. Minola, G. Dellea, M. Le Tacon, M. Moretti Sala, C. Morawe, J.-Ch. Peffen, R. Supruangnet, F. Yakhou, G. Ghiringhelli, and N. B. Brookes, Rev. Sci. Instrum. 85, 115104 (2014).

[34] M. Minola, G. Dellea, H. Gretarsson, Y. Y. Peng, Y. Lu, J. Porras, T. Loew, F. Yakhou, N. B. Brookes, Y. B. Huang, J. Pelliciari, T. Schmitt, G. Ghiringhelli, B. Keimer, L. Braicovich, and M. Le Tacon, Phys. Rev. Lett. 114, 217003 (2015).

[35] S. Sugai, H. Suzuki, Y. Takayanagi, T. Hosokawa, and N. Hayamizu, Phys. Rev. B 68, 184504 (2003).

[36] J. Graf, M. d'Astuto, C. Jozwiak, D. R. Garcia, N. L. Saini, M. Krisch, K. Ikeuchi, A. Q. R. Baron, H. Eisaki, and A. Lanzara, Phys. Rev. Lett. 100, 227002 (2008).

[37] Y. Y. Peng, J. Q. Meng, L. Zhao, Y. Liu, J. F. He, G. D. Liu, X. L. Dong, S. L. He, J. Zhang, C. T. Chen, Z. Y. Xu, and X. J. Zhou, Chin. Phys. Lett. 30, 067402 (2013).

[38] L. Braicovich et al. (unpublished).

[39] R. Coldea, S. M. Hayden, G. Aeppli, T. G. Perring, C. D. Frost, T. E. Mason, S.-W. Cheong, and Z. Fisk, Phys. Rev. Lett. 86, 5377 (2001). 
[40] D. Reznik, Physica C 481, 75 (2012).

[41] W. D. Wise, K. Chatterjee, M. C. Boyer, T. Kondo, T. Takeuchi, H. Ikuta, Z. J. Xu, J. S. Wen, G. D. Gu, Y. Wang, and E. W. Hudson, Nat. Phys. 5, 213 (2009).

[42] M. Le Tacon, A. Bosak, S. M. Souliou, G. Dellea, T. Loew, R. Heid, K.-P. Bohnen, G. Ghiringhelli, M. Krisch, and B. Keimer, Nat. Phys. 10, 52 (2013).
[43] E. Blackburn, J. Chang, A. H. Said, B. M. Leu, Ruixing Liang, D. A. Bonn, W. N. Hardy, E. M. Forgan, and S. M. Hayden, Phys. Rev. B 88, 054506 (2013).

[44] M. P. M. Dean, A. J. A. James, A. C. Walters, V. Bisogni, I. Jarrige, M. Hucker, E. Giannini, M. Fujita, J. Pelliciari, Y. B. Huang, R. M. Konik, T. Schmitt, and J. P. Hill, Phys. Rev. B 90, 220506 (2014). 